\title{
IMMUNOHISTOPATHOLOGICAL EVALUATION OF HEPATIC CD14 EXPRESSION IN MURINE SCHISTOSOMIASIS MANSONI BY
}

\author{
TAREK ABOUSHOUSHA ${ }^{1 *}$, CHRISTINA ATEF GHALY ${ }^{2^{* *}}$, MOHAMED GALAL ${ }^{2}$ \\ And AMIRA KAMEL ${ }^{1}$
}

Department of Pathology ${ }^{1}$, Theodor Bilharz Research Institute, Imbaba P. O. Box 30, and Faculty of Biotechnology ${ }^{2}$, October University for Modern Sciences and Arts (MSA), Giza, Egypt ("t.aboushousha@tbri.gov.eg, ${ }^{* *}$ christina.atef@msa.edu.eg)

\begin{abstract}
Bilharziasis is one of the most common parasitic diseases, mostly affecting the liver by causing the formation of granuloma and hepatic fibrosis, and historically endemic diseases in Egypt. Hundreds of studies examined the hepatic schistosomiasis pathogenesis to find out points for possible drug interference with disease progression and complications. The macrophages are the major source of CD14 in liver granulomas of S. mansoni-infected mice and CD14 might have regulatory roles during infection. The current study evaluated the cellular expression of CD14 in livers of $S$. mansoni infected mice in comparison with healthy controls, for assessment of its potential regulatory role in schistosome-induced hepatic fibrosis. Histopathological study of livers of $S$. mansoni infected mice after 12 weeks of infection was conducted using routine H\&E stain and M.T stain for tissue fibrosis. Immunohistochemical evaluation of CD14 positive cells was also performed using DAB as a chromogen. The results showed upregulation of CD14 expression in infected mice, compared to healthy control mice, with variable percentage and different distribution in relation to granuloma type.
\end{abstract}

Key words: Egypt, CD14, Schistosomiasis mansoni, Liver, Immunohistochemistry, EMT

\section{Introduction}

Schistosomiasis is a waterborne disease caused by a blood fluke results from parasitization by worms of the genus Schistosoma; S. haematobium, S. japonicum, and S. mansoni are the most widespread species (Tang et al, 2018). Bilharziasis is one of the commonest parasitic diseases, mostly affecting the liver by causing granuloma and hepatic fibrosis, associated with severe morbidity (Dahesh and Farid, 2016). Schistosomiasis excessively affects people who have limited access to potable water and sanitation living in the tropics and subtropics 240 million people are infected, with 700 million people at risk of getting infected and estimated to be 50,000-100,000 died annually (Dkhile et al, 2015). Schistosoma-induced liver injury results from a granulomatous inflammatory reaction around trapped Schistosoma eggs in presinusoidal periportal spaces. In early infection, a predominantly hypercellular nonfibrotic granuloma response produces liver dysfunction that is not clinically detectable. Imaging studies may reveal enlargement of the left liver lobe without changes in the liver parenchyma or splenomegaly. Reversibility of these findings were expected in 12 months after chemotherapy. Development of chronicity in collagen deposition in the periportal spaces was the basis of the pathognomonic pathological feature of schistosomal associated liver fibrosis or Symmers' pipestem fibrosis (Cavalcanti et al, 2015).

The expression evaluation of pattern recognition receptors (PRRs) on Antigen-presenting cell (APCs) in mice infected with the helminth parasite Schistosoma mansoni observed an upregulation of CD14 expression on macrophages (Pearce et al, 2004). The highest expression of CD14 was found on liver macrophages in infected mice. Collectively, these data show the role for CD14 in regulating macrophage plasticity and CD4 T cell biasing during helminth infection. There was also an increased recruitment of alternatively activated cells in livers of CD14deficient mice. This suggested that CD14 might have regulatory roles during infection and that macrophages are the major source 
of CD14 in liver granulomas of $S$. mansoniinfected mice (Tundup et al, 2014).

Granulocytes $(\mathrm{CD} 11 \mathrm{~b}+)$ are recruited to egg granulomas in livers of infected mice, in contrast to what was in the livers of uninfected mice. Macrophages, eosinophils, neutrophils, and some lymphocytes are present in liver egg granulomas of $S$. mansoni-infected mice (Pearce and MacDonald, 2002). Therefore, to analyze the expression of CD14 on granulocytes in liver egg granulomas, prepared single-cell suspensions of liver granulomas and then stained them with surface markers against macrophages, eosinophils, and neutrophils as well as CD14. They found that only CD11granulocytes had high levels of CD14 expression, compared to granulocytes with low expression of CD11b (CD11b+). The experiments showed that CD14 expression was highly upregulated in schistosome-infected (Tundup et al, 2014).

CD14-deficient mice were associated with reduced egg burdens in the livers of infected mice compared to wt controls, suggesting that immune effector cells and/or mediators regulated via CD14 may play roles in parasite fecundity. CD14 may regulate M1 and M2 phenotypes in livers of $S$. mansoniinfected mice. CD14 expression by macrophage is a key regulator of macrophage M1/M2 plasticity. This is supported by earlier observations (Herbert et al, 2004).CD14 may help in recognition of glycolipids as well as dsRNAs in controlling macrophage M1 activation and immune responses during infection (Baumann et al, 2010). In additional studies examining the role of CD14 in the regulation of immune responses need to be performed before such a hypothesis can be confirm (Lee et al, 2006).

The present work aimed to monitor the role of CD14 in macrophage activation, pathology, and immune responses in vivo during helminth infection. Given that activation and recruitment of macrophages are dominant features of many inflammatory diseases, such as cancer and autoimmune, cardio- vascular, metabolic, and allergic diseases, CD14 may serve as a potential, broadly immunotherapeutic target for numerous proinflammatory diseases.

\section{Materials and Methods}

Ethics statement: The guide for the Care and Use of Laboratory Animals was in accordance with the American Association for Accreditation of Laboratory Animal Care (AALAC) and the Animal Welfare Act (AWA). The study was approved by Theodor Bilharz Research Institute Ethical committee.

Mice, parasites, and infection: C57BL/6 female albino mice were housed at the animal house (Theodor Bilharz Research Institute) under specific-pathogen-free conditions. Two groups of mice (10 for each) were segregated in special cages, fed with conventional food pellets and drinking tap water. G1 constituted Egyptian strain of $S$. mansoni infected mice. G2 represented healthy control (non-infected) mice. G1 mice were subcutaneous infected with 50 to 60 cercariae. They were sacrificed at 12 weeks post infection, livers were removed for histopathological and immunohistochemistry studies

Paraffin sections from hepatic lesions undergo de-paraffinization, rehydration. Endogenous peroxidase was blocked with methanol containing 3\% hydrogen peroxide. Antigen retrieval was performed by microwaving the sections in citrate buffer, $\mathrm{pH}$ 6.0. Sections were incubated overnight at $4^{\circ} \mathrm{C}$ in humid chamber with the primary antibodies: Anti-CD14 (monoclonal antibody, Beckman Coulter, clone RMO52). Next day, sections washed twice in PBS, then the second biotinated antibody was applied for 20min, washed, followed by application of Envision detection system (Universal Detection Kit, Dako, Denmark). Antigen was localized by adding 3, 3'diaminobenzidine tetrahydrochloride (DAB) substrate chromogen solution. Slides were counterstained with hematoxylin, dehydrated in ethanol and mounted. 
For each setting, positive and negative control slides were included. As a negative control, hepatic tissue was processed in mentioned sequences but without primary antibodies instead adds non-immune immunoglobulin G (IgG; DAKO, Glostrup, Copenhagen, Denmark).

Paraffin sections were stained by hematoxylin and eosin for histopathological examination, counting \& size of granulomas, as well as identification of inflammatory and other types of cells. Counting of granulomas was done in 5 successive low power (100X) fields. Granuloma size was represented as the mean diameter of each granuloma with central ova, non-amalgamated and away from the portal tract. This was dome through the software (VISION) supplied by Zeiss Company. Liver sections were also stained by Masson's Trichrome stain, to assess hepatic fibrosis and identification of types of granuloma (cellular or fibrocellular).

Interpretation of immunostaining and scoring analysis: Hepatic sections were blindquantified by two pathologists. Sections were examined by light microscope and. photographs were taken using a microscope- camera (Axio-Cam, MRc5, Zeiss, Germany). CD14 positive cells were identified by cytoplasm brown coloration. Cells with these criteria were matched with corresponding cells in stained sections to identify their nature. CD14 positive cells were counted with other inflammatory cells \%. Localization of CD14 positive cells were in control and in $S$. mansoni infected livers.

Statistical Study: Data were processed on SPSS program version 20. Comparison between means were done by student T-test, with a significant values with $(\mathrm{p}<0.05)$.

\section{Results}

Histopathological examination of liver sections from infected mice revealed many lobular and portal egg-granulomas, surrounded by cellular or fibrocellular tissue response. There was expansion of portal tracts by inflammatory cells with vascular proliferation and fibrous tissue deposition. Hepatic lobule showed Kupffer cell hyperplasia, pigmentation, increase in sinusoidal monocytes and mild sinusoidal fibrosis. These were absent in liver sections of healthy controls. Only few mononuclear cells were detected in some portal tracts.

Table 1: Control and S. mansoni infected mice as to granuloma count, granuloma size \& $\mathrm{CD} 14^{+}$cellular expression:

\begin{tabular}{|c|c|c|c|c|c|}
\hline \multicolumn{2}{|l|}{ group } & Granuloma count & Granuloma Size & $\mathrm{CD}_{14} 4^{+}$cells & Localization of CD14 ${ }^{+}$cells \\
\hline & & $\begin{array}{l}\text { Mean in } 5 \text { succes- } \\
\text { sive LPF }\end{array}$ & $\begin{array}{l}\text { Mean diameter } \\
(\mu \mathrm{m})\end{array}$ & Mean percentage & $\begin{array}{c}\text { (+++): Mostly } \\
(++): \text { moderately } \\
(+) \text { : Infrequently } \\
\end{array}$ \\
\hline \multirow{2}{*}{$\begin{array}{l}\text { Control (10 } \\
\text { mice) }\end{array}$} & Mean & 0.0 & - & 3.50 & \multirow[t]{2}{*}{ Portal tracts $(+)$} \\
\hline & S.D. & 0.0 & - & 1.19 & \\
\hline \multirow{2}{*}{$\begin{array}{l}\text { Infected (10 } \\
\text { mice) }\end{array}$} & Mean & 66.18 & 171.63 & 7.09 & \multirow{2}{*}{$\begin{array}{c}\text { Granulomas }(+++) \\
\text { Portal tracts }(++) \\
\text { Lobular }(+)\end{array}$} \\
\hline & S.D. & 3.15 & 35.53 & 2.54 & \\
\hline P Value & & H.S. & - & H.S & \\
\hline
\end{tabular}

S.D.: Standard Deviation

H.S: High significance $(\mathrm{p}<0.0001)$

$\mathrm{CD} 14^{+}$cells were infrequently recognized within portal tracts of control mice, while presented at peripheral zones of egg granulomas and in portal

tracts and hepatic lobules of $S$. mansoni infected mice. Difference in $\mathrm{CD} 14^{+}$cellular expression between both was highly significant $(\mathrm{p}<0.0001)$. Table 2: Difference in \% and localization of $\mathrm{CD}_{14}{ }^{+}$cells in cellular \& fibrocellular granulomas of infected mice:

\begin{tabular}{|l|c|c|c|c|}
\hline Granuloma Type & $\begin{array}{c}\text { Granuloma count } \\
\text { (in 5 LPF) }\end{array}$ & $\begin{array}{c}\text { Granuloma } \\
\text { Diameter }(\mu \mathrm{m})\end{array}$ & $\begin{array}{c}\text { Percentage of } \\
\text { CD14 }{ }^{+} \text {cells }\end{array}$ & \multirow{2}{*}{$\begin{array}{c}\text { Localization of } \\
\text { Granulomatous } \\
\text { CD14 }\end{array}$} \\
\cline { 2 - 3 } cells
\end{tabular}

S.D.: Standard Deviation 
Histopathological results showed higher percentage of fibrocellular granulomas compared to cellular egg granulomas in livers of $S$. mansoni infected mice (group1) with significant difference $(\mathrm{p}<0.0001)$. Meanwhile, cellular granulomas were of larger diameters compared to fibrocellular ones, with statistically significant differences $(\mathrm{p}<0.0001)$.

The mean percentage of $\mathrm{CD} 14^{+}$cells was higher in the fibrocellular granulomas compared to cellular granulomas with significant difference $(p<0.05)$, however, as cellular granulomas have a greatly higher absolute number of inflammatory cells compared to fibrocellular ones, the absolute number of $\mathrm{CD} 14^{+}$cells is higher in cellular granulomas. On the other hand, the distribution of $\mathrm{CD} 14^{+}$ cells differs in both granuloma types; they are mostly peripheral in cellular granulomas, while these cells located both at the periphery and scattered throughout the whole fibrocellular granulomas.

\section{Discussion}

Many Studies showed that schistosomes caused small proportion of individuals presents severe clinical disease (as periportal fibrosis (PPF)) that may lead to death. Severe PPF results from an abnormal deposition of extracellular matrix proteins in the periportal spaces due to a chronic inflammation triggered by Schistosomes eggs (Zaiss et al, 2015). In the present study, histopathological examination of liver sections from infected mice revealed many lobular and portal egg-granulomas, surrounded by cellular or fibrocellular tissue response. Also, portal tracts showed expansion by inflammatory cells with vascular proliferation and fibrous tissue deposition. In infected mice, hepatic lobule showed Kupffer cell hypeplasia and pigmentation, increase in the sinusoidal monocytes and mild sinusoidal fibrosis. These features were absent in liver sections from the healthy control mice, where only few mononuclear cells were detected in some portal tracts. The present results showed that $73 \%$ of the egg-granulomas of the infected group were fibrocellu- lar while only $27 \%$ of the granulomas were cellular. Early cellular granulomas consist mostly of neutrophils with some eosinophils and lymphocytes; while later-on they consisted mostly of mononuclear inflammatory cells including macrophages at their peripheral zones. Fibrocellular granulomas consist of central zones of fibroblasts and deposited collagen fibers, surrounded by mononuclear inflammatory cells including macrophages. Previous studies showed that granulocytes $(\mathrm{CD} 11 \mathrm{~b}+)$ are recruited to egg granulomas in livers of infected mice, in contrast to what is seen in the livers of uninfected mice. In addition macrophages, eosinophils, neutrophils, and some lymphocytes are present in liver egg granulomas of $S$. mansoni-infected mice (Pearce and MacDonald, 2002). The present results showed that CD14 positive expression was infrequently recognized within monocytes in the portal tracts of the healthy control mice (group2) and occasionally within scattered hepatocytes, while there were obvious upregulation of CD14 in monocytes at the peripheral zones of egg granulomas as well as in the portal tracts and hepatic lobules of S. mansoni infected mice (group1). The difference in CD14 cellular expression between both groups is highly significant $(\mathrm{p}<0.0001)$.

In addition, the endotoxin-responsive monocytes/macrophages (CD14-positive) were potential sources of profibrogenic factors. The number of the hepatic CD14 ${ }^{+}$cells increased in advanced fibrosis in subjects with primary biliary cirrhosis and hepatitis C. In addition, the increased CD14 positive cells were associated with high inflammatory activity (Leicester et al, 2006).

In the present study, cellular granulomas were of larger diameters compared to fibrocellular ones, with statistically significant differences $(p<0.0001)$. The mean percentage of $\mathrm{CD} 14^{+}$cells was higher in fibrocellular granulomas compared to cellular granulomas with significant difference $(\mathrm{p}<0.05)$, however, as cellular granulomas have a greatly higher absolute number of inflamma- 
tory cells compared to fibrocellular ones, the absolute number of $\mathrm{CD} 14^{+}$cells is higher in cellular granulomas. Also, studies showed that liver fibrosis is a wound healing response to chronic liver injury and inflammation in which macrophages and infiltrating monocytes participate in both the development and resolution phase. In humans, three monocyte subsets were identified: the classical CD14++CD16-, intermediate CD14++ CD16+, and non-classical CD14+ CD16++ monocytes, \& intermediate CD14++ CD16+ monocytes accumulate in chronically inflamed human liver as a consequence of enhanced recruitment from blood and local differentiation from classical CD14++ CD16monocytes (Liaskou et al, 2013).

Also, the results showed $\mathrm{CD} 14^{+}$cells distribution differed in both granuloma types; they were mostly peripheral in cellular granulomas, while these cells located both at the periphery and scattered throughout whole fibrocellular granulomas Studies evaluated expression of PRRs on APCs in mice infected with $S$. mansoni and observed an up-regulation of CD14 expression on macrophages. Highest CD14expression was on liver macrophages in infected mice. These data identify a previously unrecognized role for CD14 in regulating macrophage plasticity and CD4T cell biasing during helminthiasis (Vanhoutte et al, 2007). This showed that CD14 expression increased in livers of infected mice suggested that CD14 might have regulatory roles during infection. Macrophages were CD14 major source in liver granulomas of $S$. mansoni-infected mice (Han et al, 2010). So. to analyze the expression of CD14 on granulocytes in liver egg granulomas, prepared single-cell suspensions of liver granulomas and then stained with surface markers against macrophages, eosinophils, neutrophils and CD14 found that only CD11 granulocytes had high levels of CD14 expression, compared to granulocytes with the low expression of CD11b(Tundup et al, 2014). These experiments demonstrated that CD14 expression was highly upregulated in schistosome-infected. CD14 may regulate M1 \& M2 phenotypes in livers of S. mansoni-infected mice. CD14 expression by macrophage is a key regulator of macrophage M1/M2 plasticity, which agreed with Layland et al. (2007). Role of CD14 in the regulation of immune responses need to be performed before such a hypothesis can be confirmed. CD14 may help in recognition of glycolipids as well as dsRNAs in controlling macrophage M1 activation and immune responses during infection (Sackesen et al, 2011)

Comparably, many studies showed the relationship between soluble types of CD14 (sCD14) and histological features in patients with other chronic liver diseases like non alcoholic fatty liver disease (NAFLD). Also, the increased sCD14 levels in nonalcoholic steato-hepatitis (NASH) patients were highly correlated with increased hepatic CD14 expression and liver inflammation (Ogawa et al, 2013). Polymorphism in human CD14 promoter region caused in higher levels of CD14, associated with an increase in severity of alcoholic liver disease (Jarvelainen et al, 2001). Animal studies, strongly supported involvement of CD14 in alcoholic liver disease and that CD14 was a risk factor for ethanol-induced pathology (Yin et al, 2001). Up-regulation of CD14 sensitization showed that CD14 levels are elevated in liver following chronic exposure to ethanol (Kono et al, 2000; Kishore et al, 2002). Also the role of CD14 in liver inflammation after chronic hepatitis B virus infection is essential for hepatocellular carcinoma (HCC) development. The individuals with HCC outcome had statistically higher serum levels of IL-23 than controls. HCC tissues analysis showed that $\mathrm{CD} 14^{+}$inflammatory macrophages were major IL-23 producers (Zang et al, 2018).

\section{Conclusions}

The outcome data proved that $\mathrm{CD} 14^{+}$cells played a regulatory role in the pathogenesis of $S$. mansoni induced liver fibrosis through modulation of the epithelial-mesenchymal transition (EMT). 
A point is the use of more tissue markers, to evaluate the effect of different anti-helminthic, anti-inflammatory and anti-fibrotic therapeutic agents on schistosomiasis or other liver diseases. Such studies are ongoing and will be published in due time elsewhere.

\section{Acknowledgment}

The authors are grateful to Prof. Dr. Ayman Diab, Dean, Faculty of Biotechnology, and Prof. Dr. Gehan Safwat, Vice Dean, who kindly supporting and facilitating this work. Thanks are also due to Staff Members of Department of Pathology, Theodor Bilharz Research Institute.

\section{References}

Dahesh, SMA, Farid, BE, 2016: Epidemiological situation of urinary schistosomiasis in Tamwah Area, Giza, Egypt: assessment and control. J. Egypt. Soc. Parasitol. 46, 3:485-96.

Dkhil, MA, Bauomy,AA, Diab, MSM, AlQuraishy, S, 2015: Antioxidant and hepatoprotective role of gold nanoparticles against murine hepatic schistosomiasis. Inter. J, Nanomed. 10: 7467-75.

Baumann, CL, Aspalter, IM, Sharif, O, PichImair, A, et al, 2010: CD14 is a coreceptor of Toll-like receptors 7 and 9. J. Exp. Med. 207: 2689-701

Cavalcanti, M, Araujo-Neto, J, Peralta, J, 2015: Schistosomiasis: Clinical Management of Liver Disease, J. Clin. Liver Dis. 6:59-62

Chevillard, C, Eboumbou, C, Nasr-Eldin, M A, Bream, E, et al, 2003: IFN__ Polymorphisms (IFN-2109 \& IFN-3810) are associated with severe hepatic fibrosis in human hepatic schistosomiasis (Schistosoma mansoni). J. Immunol. 171:5596-601.

Herbert, DR, Holscher, C, Mohrs M, Arendse, B, et al, 2004: Alternative macrophage activation is essential for survival during schistosomiasis and downmodulates $\mathrm{T}$ helper 1 responses and immunopathology. Immunity 20:623-35.

Han, D, She, W, Zhang, L, 2010: Association of CD14 gene polymorphism C-159T with allergic rhinitis. Am. J. Rhinol. Allergy 24, 1:e1-3.

Jarvelainen, HA, Orpana, A, Perola, M, Savolainen, VT, et al, 2001: Promoter polymorphism of the CD14 endotoxin receptor gene as a risk factor for alcoholic liver disease, J. Hepatol. 33, 11:48-53.

Kishore, R, Hill, JR, McMullen, M, Frenkel,
J, 2002: Nagy LE. ERK $1 / 2$ and Egr-1 contribute to increased TNF- $\alpha$ production in rat Kupffer cells after chronic ethanol feeding ,Am. J. Physiol. 282:G6-15.

Kono, H, Wheeler, MD, Rusyn, I, Lin, M, et al, 2000: Gender differences in early alcoholinduced liver injury: role of CD14, NF-kappaB, and TNF-alpha. Am. J. Physiol. 278:G652-61

Lee, HK, Dunzendorfer, S, Soldau, K, Tobias, PS, 2006: Double-stranded RNA-mediated TL R3 activation is enhanced by CD14. Immunity 24:153-63.

Liaskou, E, Zimmermann, HW, Li, KK, Oo, YH, 2013: Monocyte subsets in human liver diseases how distinct phenotypic and functional characteristics. Hepatology 57, 1:38-42

Layland, LE, Rad, R, Wagner, H, da Costa, C U, 2007: Immunopathology in schistosomiasis is controlled by antigen-specific regulatory $\mathrm{T}$ cells primed in the presence of TLR2. Eur. J. Immunol. 37:2174-84.

Michael, D, Wheeler, Ronald, G, Thurman, A, 2003: Up-regulation of CD14 in liver caused by acute ethanol involves oxidant-dependent AP-1 pathway, J. Biol. Chemis. 278:8435-41

Ogawa, Y, Imajo, K, Yoneda, M, Kessoku, T, et al, 2013: Soluble CD14 levels reflect liver inflammation in patients with nonalcoholic steatohepatitis. J. Plosone. 19:e65211.

Pearce, EJ, MK, C, Sun, J, McKee, AS, et al, 2004: Th2 response polarization during infection with the helminth parasite Schistosoma mamsoni. Immunol. Rev. 201:117-26.

Pearce, EJ, MacDonald, AS, 2002: The immunobiology of schistosomiasis. Nat. Rev. Immunol. 2:499-51

Sackesen, C, Birben, E, Soyer, OU, Sahiner, UM, et al, 2011: The effect of CD14 C159T polymorphism on in vitro IgE synthesis and cytokine production by PBMC from children with asthma. J. Allergy 66:48-57.

Tang, C, Liu, Z, Gao, Y, Xiong, F, 2018: Schistosoma infection and Schistosoma-derived products modulate the immune responses associated with protection against type 2 Diabetes. J. Frontiers 8:1990

Tundup, S, Srivastava, L, Nagy, T, Harna, D, 2014: CD14 influences host immune responses and alternative activation of macrophages during Schistosoma mansoni infection. JASM 3:240-51. Vanhoutte, F, Breuilh, L, Fontaine, J, Zouain, CS, et al, 2007: Toll-likereceptor (TLR)2 and TLR3 sensing is required for dendritic cell acti- 
vation, but dispensable to control Schistosoma mansoni infection and pathology. J. Microbes Infect. 9:1606-13.

Yin, M, Bradford, BU, Wheeler, MD, Uesugi, T, et al, 2001: Reduced early alcohol-induced liver injury in CD14-deficient mice. J. Immunol. 166:4737-42

Zang, M, Li, Y, He, H, Ding, H, et al, 2018: IL-23 production of liver inflammatory macro- phages to damaged hepatocytes promotes hepatocellular carcinoma development after chronic hepatitis B virus infection. Biochim. Biophys. Acta. Mol. Basis Dis. 1864, 12:3759-70.

Zaiss, MM, Rapin, A, Lebon, L, Dubey, LK, et al, 2015: The intestinal microbiota contributes to the ability of helminths to modulate allergic inflammation. J. Immunity 43, 5:998-1010.

\section{Explanation of figures}

Fig. 1: Sections in liver of healthy control mice (G2); showed preserved hepatic architecture and unremarkable pathological changes of liver parenchyma and portal tracts (a) H \& E stain, X100; (b) Masson trichrome stain X100; (c) Few hepatocytes and mononuclear cells showed positive expression for CD14 antigen (arrows). $73 \%$ of granulomas of infected mice were fibrocellular with only $27 \%$ of granulomas cellular. Early cellular granulomas consist mostly of neutrophils with some eosinophils and lymphocytes, later on they consist mostly of mononuclear inflammatory cells including macrophages at peripheral zones. Fibrocellular granulomas of central fibroblasts zones, and deposited collagen fibers, surrounded by mononuclear inflammatory cells including macrophages.

Fig. 2: Sections in livers of $S$. mansoni infected mice showed distorted hepatic architecture by many egg-granulomas (a\&c)H\&E stain, X200; (b\&d)MT stain, X200, with upregulation of CD14 expression in egg-granulomas(e) and portal tracts (f)

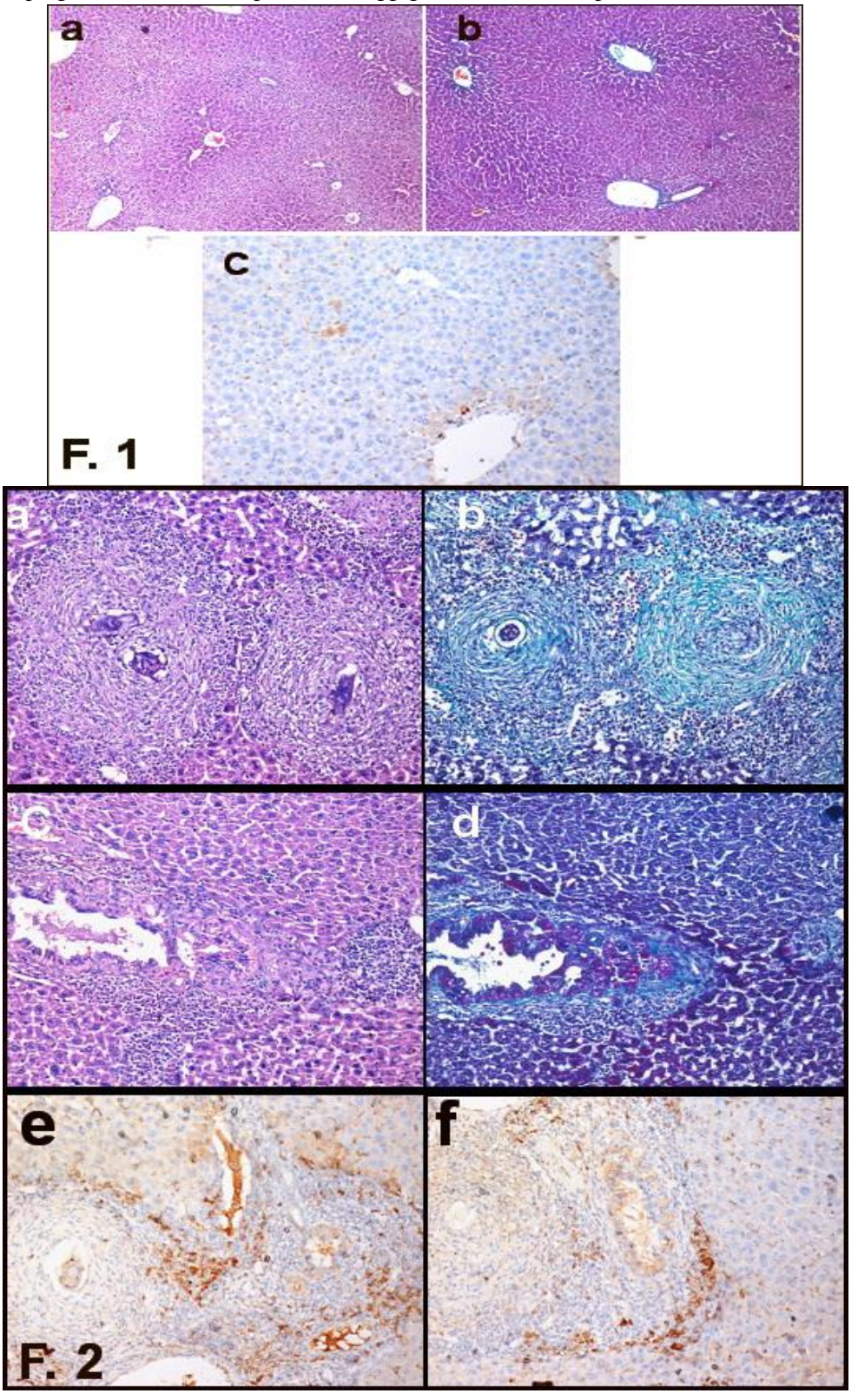

